Correction

\title{
Correction: The different pathogenesis of sporadic adenoma and adenocarcinoma in non-ampullary lesions of the proximal and distal duodenum
}

\author{
Ayumi Niwa ${ }^{1, *}$, Seiya Kuwano ${ }^{1, *}$, Hiroyuki Tomita ${ }^{1}$, Keita Kimura ${ }^{1}$, Yukiya Orihara ${ }^{1}$, \\ Tomohiro Kanayama ${ }^{1}$, Kei Noguchi ${ }^{1}$, Kenji Hisamatsu ${ }^{1}$, Takayuki Nakashima ${ }^{1}$, \\ Yuichiro Hatano ${ }^{1}$, Akihiro Hirata², Tatsuhiko Miyazaki³, Kazuhiro Kaneko4, Takuji \\ Tanaka $^{5}$ and Akira Hara ${ }^{1}$ \\ ${ }^{1}$ Department of Tumor Pathology, Gifu University Graduate School of Medicine, Gifu, Japan \\ 2 Division of Animal Experiment, Life Science Research Center, Gifu University, Gifu, Japan \\ 3 Division of Pathology, Gifu University Hospital, Gifu, Japan \\ ${ }^{4}$ Department of Gastroenterology, Endoscopy Division, National Cancer Center Hospital East, Kashiwa, Japan \\ ${ }^{5}$ Department of Diagnostic Pathology (DDP) and Research Center of Diagnostic Pathology (RC-DiP), Gifu Municipal Hospital, \\ Gifu, Japan \\ * These authors have contributed equally to this work
}

Published: February 16, 2018

Copyright: Niwa et al. This is an open-access article distributed under the terms of the Creative Commons Attribution License 3.0 (CC BY 3.0), which permits unrestricted use, distribution, and reproduction in any medium, provided the original author and source are credited.

This article has been corrected: the title has been corrected.

Original article: Oncotarget. 2017; 8:41078-41090. https://doi.org/10.18632/oncotarget.17051 